\title{
QUANTIFICAÇÃO DE ADITIVOS DETERGENTES E DISPERSANTES EM GASOLINAS POR TERMOGRAVIMETRIA
}

\author{
Amanda Pereira Franco dos Santos ${ }^{1}$, Jo Dweck ${ }^{2}$ e Luiz Antonio d'Avila ${ }^{1}$ \\ ${ }^{1}$ Laboratório de Combustíveis e Derivados de Petróleo - LABCOM, Escola de Química, \\ Universidade Federal do Rio de Janeiro \\ ${ }^{2}$ Laboratório de Análises Térmicas - LABAT, Escola de Química, Universidade Federal do \\ Rio de Janeiro
}

E-mails: apfranco@,eq.ufrj.br, dweck@eq.ufrj.br, davila@eq.ufrj.br

\section{RESUMO}

Embora a gasolina do tipo $\mathrm{C}$ aditivada já seja comercializada no Brasil há muito tempo, não existe nenhum método de referência estabelecido pela Agência Nacional de Petróleo, Gás Natural e Biocombustível (ANP), que possibilite a análise quantitativa dos aditivos detergentes dispersantes nela presentes. Isto é dificultado pelo desconhecimento da composição exata dos aditivos, tratados como segredos industriais pelos fabricantes, comercializados sob a forma de pacotes de aditivos. Este trabalho propõe um método de quantificação, somente por termogravimetria, precedido apenas de pré-concentração dos aditivos pelo ensaio rotineiro de destilação atmosférica. Foram analisadas seis amostras de resíduos de destilação de gasolinas aditivadas com o aditivo "A" nas concentrações nominais de 100 à $5000 \mathrm{mg} \cdot \mathrm{kg}^{-1}$, além de gasolina sem aditivo (branco). A partir da taxa de concentração do aditivo obtida na destilação atmosférica e da perda de massa referente aos seus princípios ativos encontrados na $\mathrm{TG}$, foi possível quantificar os aditivos detergentes dispersantes presentes na gasolina, com erro relativo médio de até $9 \%$. $O$ método desenvolvido é mais simples e de menor custo dos até então propostos na literatura e os resultados apontam como potencialmente adequado para análises de quaisquer gasolinas aditivadas, independentemente dos seus pacotes de aditivos e aplicável pelos laboratórios de controle de qualidade de combustíveis.

\section{INTRODUÇÃO}

A Resolução ANP N 40 de 25 de outubro de 2013 estabelece as especificações das gasolinas de uso automotivo e as obrigações quanto ao controle da qualidade a serem atendidas pelos diversos agentes econômicos que comercializam o produto em todo o território nacional. As gasolinas automotivas brasileiras podem ser do tipo comum ou premium e classificam-se em gasolina $\mathrm{A}$, isenta de componentes oxigenados e gasolina $\mathrm{C}$, obtida da mistura de gasolina $\mathrm{A}$ e etanol anidro combustível. A diferença entre as gasolinas comum e premium é o valor mínimo do índice antidetonante (resistência à detonação), respectivamente, 87 e 91 [1].

Além disso, há a gasolina $\mathrm{C}$ aditivada com aditivos detergentes dispersantes, que melhoram o desempenho do motor, mantendo os sólidos em suspensão e finamente dispersos, evitando que se depositem em locais indesejáveis e também reduzem a emissão de poluentes [1] e [2]. 
A gasolina aditivada se diferencia visualmente da comum pela sua coloração, pois enquanto a comum varia de incolor a amarelada, a aditivada recebe um corante que pode ser de qualquer cor (geralmente verde), com exceção de azul (reservada para a gasolina de aviação) [1] e [3]. $\mathrm{O}$ efeito do corante limita-se à diferenciação dos dois tipos de produto e não interfere em sua funcionalidade. Nem sempre a adição do corante é garantia de adição do aditivo com propriedades detergentes dispersantes.

Os aditivos são produtos adicionados tanto para adequar o combustível às especificações técnicas (ex. aditivos antidetonantes) quanto para melhorar as características de desempenho e ambientais dos combustíveis convencionais (ex. aditivos detergentes dispersantes) [3] e [4].

A formação e acúmulo de depósitos em válvulas de admissão durante o funcionamento de um motor de combustão interna é um problema frequente que tem como consequências a diminuição da eficiência do motor, uma vez que esses depósitos restringem o fluxo de arcombustível na câmara de combustão [5], com consumo excessivo do combustível e o aumento das emissões de poluentes [2] e [6]. Os aditivos detergentes dispersantes tanto removem os depósitos previamente formados (aditivos clean-up) quanto minimizam a sua formação (aditivos keep-clean).

Os aditivos detergentes são substâncias que agem através dos grupos terminais polares, geralmente contendo nitrogênio, que aderem às superfícies metálicas, formando filmes protetores que previnem a formação de resíduos nas superfícies internas do motor. Os aditivos dispersantes contribuem para a dispersão de partículas que entram no motor via ar, combustível ou lubrificante, mantendo-os sólidos em suspensão e finamente dispersos, prevenindo assim a formação de depósito [7].

Além dos benefícios obtidos com o uso dos aditivos detergentes dispersantes no motor do carro, os outros benefícios do uso desses compostos incluem a diminuição do consumo de combustível em 3\%, a redução de 60 a $70 \%$ de depósitos, uma redução de 50 a $60 \%$ das emissões de monóxido de carbono e hidrocarbonetos e de $20 \%$ de óxidos de nitrogênio. Por sua vez, isso permite: aumentar a confiabilidade do funcionamento do sistema de combustível; prolongar o tempo de vida do motor; reduzir os custos de manutenção; reduzir a formação de fuligem na câmara de combustão e, assim, diminuir os requisitos do motor para o número de octanas da gasolina [2] e [8].

Aditivos para combustíveis são produtos constituídos por um ou mais componentes ativos com ou sem diluente. O componente ativo de um aditivo é um constituinte que melhora as propriedades do combustível automotivo e o diluente é, geralmente, o solvente que adicionado ao componente ativo facilita a sua solubilização no combustível automotivo. Segundo Silin et al. (2012), Danilov (2015), Colaiocco \& Lattanzio (1995) e Grapner et al. (2005), os pacotes de aditivos detergentes são compostos pelos seus componentes ativos, tais como aminas, amidas, amino amidas, alquenil succinimidas, polibutenos de amidas, dissolvidos em óleos minerais [4], [6], [9] e [10].

Embora a gasolina do tipo $\mathrm{C}$ aditivada já seja comercializada no Brasil há muito tempo, não há nenhum método de referência que possibilite a análise quantitativa dos aditivos detergentes dispersantes nela presentes. Uma das dificuldades é o desconhecimento da sua exata composição já que, por serem segredos industriais, são comercializados pelos fabricantes sob 
a forma de pacotes de aditivos. A quantificação desses aditivos na gasolina tem sido proposta através da: espectroscopia vibracional (FT-IR e FT-NIR) associada a técnicas quimiométricas [7] e [11] e pré-concentração dos combustíveis; ring oven para pré-concentração dos aditivos seguida de análises das imagens hiperespectrais na região do infravermelho próximo [12]; cromatografia líquida associada à termogravimetria [13], [14] e [15] e espectrometria de massas [16] e espectroscopia por ressonância magnética nuclear [17], em geral dependentes de curvas de calibração de alguns aditivos disponíveis.

1.1. Metodologias desenvolvidas pelos autores para quantificação de aditivos detergentes dispersantes em gasolinas

\subsubsection{Quantificação de aditivos detergentes dispersantes em combustiveis e lubrificantes em campo}

Foi desenvolvido um método simples, rápido, de fácil execução e seguro para identificação de aditivos detergentes dispersantes em combustíveis e óleos lubrificantes em campo por meio da diferença de comportamento cromatográfico. Isto porque os aditivos detergentes dispersantes são polares e têm natureza básica, enquanto os combustíveis (gasolina e óleo diesel) e os óleos base lubrificantes têm natureza essencialmente hidrocarbônica [18].

\subsubsection{Quantificação de aditivos detergentes dispersantes em gasolina por cromatografia de exclusão por tamanho (CET)}

Foi desenvolvido um método para quantificação de aditivos detergentes dispersantes em gasolina, a partir de sua pré-concentração por meio da destilação atmosférica (método ASTM D86) seguida da análise por cromatografia de exclusão por tamanho (CET), com detector convencional de índice de refração [13]. A determinação da curva de destilação de gasolinas (ASTM D86) é mandatória e realizada rotineiramente no monitoramento da qualidade deste combustível [19]. Este processo leva ao aumento de sua concentração no resíduo, na medida em que o volume total é reduzido de $100 \mathrm{ml}$ para cerca de $5 \mathrm{ml}$, facilitando a sua detecção [13].

De acordo com Lin et al. (2002), os aditivos devem permanecer estáveis a cerca de $250{ }^{\circ} \mathrm{C}$ e apresentar uma decomposição limpa (sem resíduos) acima da temperatura de operação do motor, que é em torno de $300{ }^{\circ} \mathrm{C}$ [20]. Em consequência, os aditivos presentes nas gasolinas aditivadas permanecem no resíduo da destilação, em função de sua menor volatilidade, devido ao seu maior peso molecular médio, polaridade e estabilidade térmica.

O método apresentou limitação, pois foi observado que os aditivos $\mathrm{G}$ e W se comportaram de maneira semelhante (coeficientes angulares muito parecidos), porém diferentes do aditivo T. Desta forma, é fundamental saber, anteriormente à análise de CET, qual aditivo foi adicionado à gasolina, para então quantificá-lo com exatidão, utilizando a curva analítica apropriada [13]. 
A questão crucial no desenvolvimento de método analítico visando determinar a concentração de aditivos detergentes dispersantes em gasolinas reais por CET é que o analista não teria conhecimento da natureza do aditivo presente, tendo, portanto necessidade de identificá-lo previamente para que seja possível sua quantificação a partir das respectivas curvas analíticas.

\subsubsection{Quantificação de aditivos detergentes dispersantes em gasolina por cromatografia de exclusão por tamanho e termogravimetria}

Foram obtidos resultados satisfatórios na caracterização do tipo de aditivo adicionado à gasolina, utilizando a termogravimetria (TG) e a termogravimetria derivada (DTG) e posterior quantificação por CET [14] e [15].

Pimentel et al. (2015), através da espectroscopia de infravermelho próximo com utilização de modelo de mínimos quadrados parciais (PLS), também observaram as mesmas semelhanças e diferenças entre os aditivos "G e W" e "T e Y" [12]. Os espectros de massas dos aditivos estudados por Silva (2017) mostraram que os aditivos "G e W" apresentam majoritariamente duas séries homólogas enquanto que o aditivo " $T$ " apenas uma [16].

Utilizando análises termogravimétricas (TG), foi demonstrado que o uso da análise termogravimétrica derivada (DTG) possibilita a identificação prévia do tipo de aditivo adicionado à gasolina, possibilitando verificar que os aditivos " $\mathrm{G}$ e $\mathrm{W}$ " tinham mesmo princípio ativo, assim como os aditivos "T e Y", permitindo sua quantificação exata por CET, visto que era possível escolher a curva analítica apropriada para cada amostra analisada.

Este trabalho, aplicado a misturas de gasolina não aditivada com diferentes concentrações de um aditivo, denominado $\mathrm{A}$, propõe um método de quantificação de aditivos detergentes e dispersantes, somente por termogravimetria, precedido apenas de pré-concentração dos aditivos pelo ensaio rotineiro de destilação atmosférica, segundo a norma ASTM D86.

\section{MATERIAIS E MÉTODOS}

Foram feitas misturas de uma gasolina comercial não aditivada, com diferentes concentrações de um aditivo A, $\left(100,200,500,1000,2500\right.$ e $5000 \mathrm{mg} \cdot \mathrm{kg}^{-1)}$, sendo aplicado a posteriori, o método abaixo desenvolvido. Os resultados obtidos foram comparados com os teores conhecidos do aditivo em cada mistura para avaliação da exatidão do método.

Após proceder a destilação atmosférica das diversas amostras das misturas preparadas, os resíduos de destilação assim obtidos para pré-concentração dos aditivos, foram analisados por termogravimetria (TG) e termogravimetria derivada (DTG) para quantificação dos aditivos. 
O equipamento utilizado foi o de análise simultânea por TG/DTA/DSC da TA Instruments, modelo Q600. Usou-se amostras com massas da ordem de $7 \mathrm{mg}$, colocadas em cadinhos de alumínio selados do tipo Tzero, com tampas micro furadas a laser. As análises foram realizadas com razão de aquecimento de $10{ }^{\circ} \mathrm{C} \cdot \mathrm{min}^{-1}$, com vazão de $100 \mathrm{~mL} \cdot \mathrm{min}^{-1}$ de nitrogênio 99,999\%, como gás de purga [21] e [22]. As curvas TG e DTG foram obtidas e analisadas usando o software de processamento de dados Universal Analysis, da TA instruments.

A diferença entre as massas percentuais residuais, medidas nas respectivas curvas TG, entre os limites dos respectivos picos DTG de perda do princípio ativo do aditivo, corresponde à sua massa percentual no resíduo de destilação. A partir da taxa de concentração do aditivo ocorrida na destilação atmosférica e do teor de princípio ativo do aditivo A, obtido por TG do aditivo, a concentração de aditivo detergente dispersante em dada mistura de gasolina aditivada, é dada pela Equação 1 :

$$
C_{a d}=\left(\frac{\left(\frac{P_{m}}{P A} x 100\right)}{T_{x}}\right) \times 10000
$$

Equação 1

onde,

$\mathrm{C}_{\mathrm{ad}}$ = concentração de aditivo presente na gasolina, $\left(\mathrm{mg} \cdot \mathrm{kg}^{-1}\right)$;

$\mathrm{P}_{\mathrm{m}}=$ perda de massa correspondente ao PA observado na TG do resíduo de destilação, $(\mathrm{m} \%)$;

$\mathrm{PA}=$ teor de princípio ativo presente no pacote de aditivo, $(\mathrm{m} \%)$;

$\mathrm{T}_{\mathrm{x}}=$ taxa de concentração do aditivo no resíduo da destilação atmosférica.

\section{RESULTADOS E DISCUSSÃO}

Na Figura 1 são apresentadas as curvas TG e DTG obtidas para a amostra de aditivo puro, denominado A, mostrando comportamento típico das análises térmicas dos aditivos detergentes dispersantes. Os resultados indicam a existência de duas etapas de perda de massa bem específicas, em faixas de temperatura bem distintas. Na primeira etapa, que ocorre até temperaturas em torno de $300^{\circ} \mathrm{C}$, ocorre a volatilização de possíveis solventes e a segunda de volatilização e/ou craqueamento dos agentes ou produtos ativos dos respectivos aditivos, a qual ocorre em faixa de temperatura bem superior $\left(300\right.$ a $\left.500^{\circ} \mathrm{C}\right)$, indicando tratar-se da decomposição da fração de maior peso molecular. Segundo Colaiocco \& Lattanzio (1995), Silin et al. (2012) e Danilov (2015), os pacotes de aditivos detergente são compostos pelo seu princípio ativo (peso molecular elevado), que pode conter aminas, amidas, amino amidas, alquenil succinimidas, polibutenos de amidas entre outros, dissolvidos em óleos minerais. Isto explica a existência de duas etapas de perda de massa na análise térmica mostrada na Figura 1 [4], [6] e [9]. 


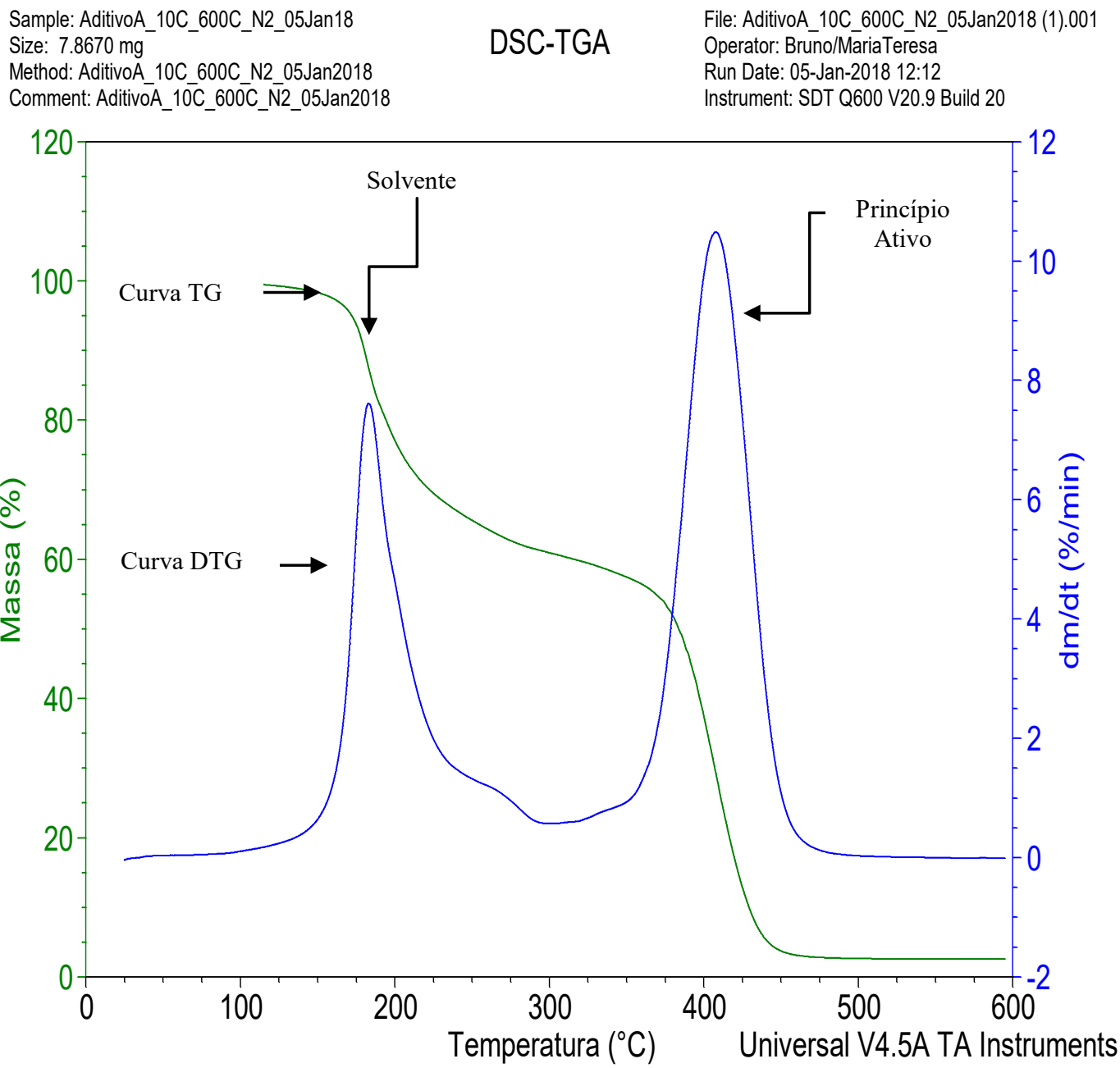

Fig 1. Curvas TG e DTG do aditivo A puro.

Para os cálculos da análise de exatidão, nas amostras de verificação com o aditivo $\mathrm{A}$, foi utilizado o percentual de 55,65 \% (valor médio obtido em triplicada), obtido pelas curvas TG e DTG da Figura 1. Cabe notar que as curvas TG/DTG deste aditivo foram muito semelhantes às dos aditivos "G e W" e "T e Y", obtidas pelos autores anteriormente [15].

Como exemplo de uma análise típica, a Figura 2 apresenta as curvas TG e DTG do resíduo de destilação da gasolina com $5000 \mathrm{mg} \cdot \mathrm{kg}^{-1}$ do aditivo A, no qual pode-se observar perfil de curvas TG e DTG similar ao apresentado na Figura 1. Os resultados também indicam a existência de duas etapas de perda de massa bem específicas. A primeira etapa ocorre até 350 ${ }^{\circ} \mathrm{C}$ e refere-se a volatilização da gasolina + solvente (presente no aditivo) e a segunda etapa ocorre em faixa de temperatura superior, próximo à $400^{\circ} \mathrm{C}$, e corresponde à etapa de perda de massa do princípio ativo do aditivo A. Cabe notar que este comportamento é muito similar ao de outros aditivos analisados por TG pelos autores. 


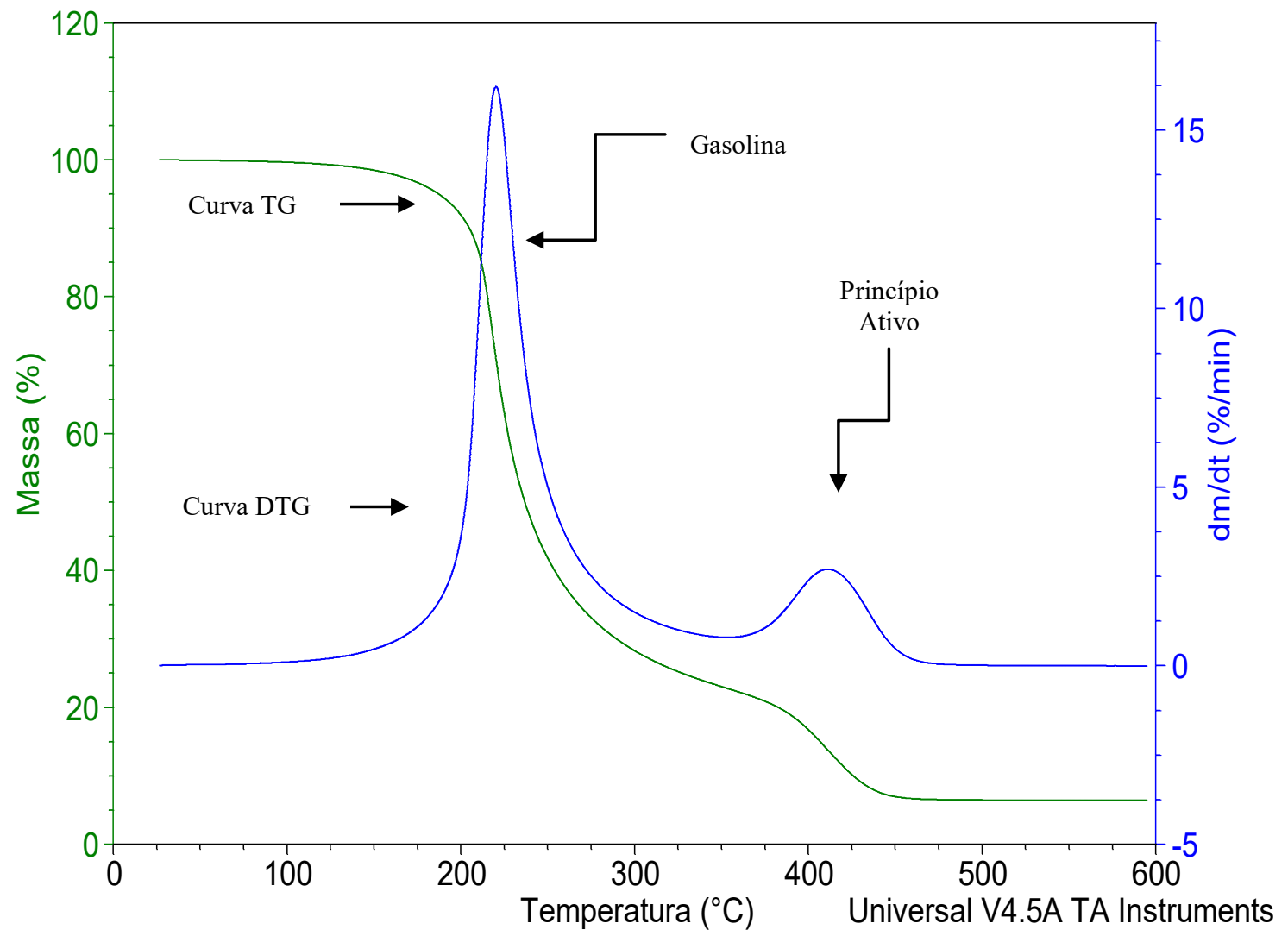

Fig 2. Curvas TG e DTG do resíduo de destilação de gasolina com $5000 \mathrm{mg} \cdot \mathrm{kg}^{-1}$ do aditivo A.

Analisaram-se os resíduos de destilação atmosférica das amostras de gasolina preparadas com aproximadamente 100, 200, 500, 1000, 2500 e $5000 \mathrm{mg} \cdot \mathrm{kg}^{-1}$ de aditivo A, conforme apresentado na Tabela 1. Observou-se que os resultados obtidos foram satisfatórios, pois o erro relativo [23] médio obtido foi de até $9,04 \%$. 
Tabela 1. Teores de aditivos detergentes dispersantes nas amostras analisadas, obtidos pelo método desenvolvido por TG.

\begin{tabular}{|c|c|c|c|c|c|c|}
\hline Amostra & $\begin{array}{l}\text { Concentração } \\
\text { real de } \\
\text { aditivo na } \\
\text { gasolina } \\
\left(\mathrm{mg} \cdot \mathrm{kg}^{-1}\right)\end{array}$ & $\begin{array}{c}\text { Perda de } \\
\text { massa do } \\
\text { PA } \\
\text { observado } \\
\text { na TG } \\
(\%)\end{array}$ & $\begin{array}{c}\text { Taxa de } \\
\text { concen- } \\
\text { tração (\%) }\end{array}$ & $\begin{array}{c}\text { Concentração } \\
\text { de aditivo na } \\
\text { gasolina } \\
\left(\mathrm{mg} \cdot \mathrm{kg}^{-1}\right)\end{array}$ & $\begin{array}{l}\text { Concentração } \\
\text { média de } \\
\text { aditivo na } \\
\text { gasolina } \\
\left(\mathrm{mg} \cdot \mathrm{kg}^{-1}\right)\end{array}$ & $\begin{array}{l}\text { Erro Relativo } \\
\text { Médio (\%) }\end{array}$ \\
\hline 100A_1a & 99,94 & 0,2914 & 51,20 & 102,27 & \multirow{2}{*}{108,97} & \multirow{2}{*}{9,04} \\
\hline 100A_1b & 99,94 & 0,3296 & 51,20 & 115,68 & & \\
\hline 200A_1a & 228,21 & 0,7288 & 63,33 & 206,79 & \multirow{2}{*}{228,24} & \multirow{2}{*}{0,01} \\
\hline $200 A \_1 b$ & 228,21 & 0,8800 & 63,33 & 249,69 & & \\
\hline 500A_1a & 500,15 & 1,7310 & 58,01 & 536,20 & \multirow{2}{*}{530,50} & \multirow{2}{*}{6,07} \\
\hline 500A_1b & 500,15 & 1,9010 & 65,09 & 524,81 & & \\
\hline $1000 A_{-} 1 \mathrm{a}$ & 999,73 & 3,4960 & 57,70 & 1088,76 & \multirow{2}{*}{1014,44} & \multirow{2}{*}{1,47} \\
\hline $1000 \mathrm{~A} \_1 \mathrm{~b}$ & 999,73 & 3,3850 & 64,70 & 940,13 & & \\
\hline $2500 A \_1 a$ & 2499,92 & 10,2200 & 62,60 & 2933,67 & \multirow{2}{*}{2635,09} & \multirow{2}{*}{5,41} \\
\hline $2500 \mathrm{~A} \_1 \mathrm{~b}$ & 2499,92 & 7,7730 & 59,78 & 2336,51 & & \\
\hline 5000A_1a & 5000,17 & 16,9200 & 54,45 & 5583,90 & \multirow{2}{*}{5340,14} & \multirow{2}{*}{6,80} \\
\hline 5000A_1b & 5000,17 & 16,2000 & 57,12 & 5096,38 & & \\
\hline
\end{tabular}

Observa-se pela Tabela 1, que o maior erro relativo entre valor obtido do teor de aditivo e o efetivamente usado na preparação da respectiva amostra de gasolina aditivada, ocorreu no caso de menor teor, embora o valor absoluto do erro médio neste caso tenha sido bem menor do que nos casos de muito maior concentração, e que poderá ser ainda mais diminuído na evolução dos trabalhos em andamento.

Por outro lado, cabe notar que o procedimento desenvolvido leva a erros aceitáveis, considerando os níveis de concentração envolvidos nas determinações, sobretudo levando-se em conta a possibilidade de uso do método no controle de qualidade de gasolinas aditivadas. Cabe notar que o teor de aditivo em uma gasolina aditivada não é atualmente considerado 
para diferenciação entre as existentes no mercado, sendo por enquanto usado apenas para sua classificação e justificativa para diferenciação de seu preço em relação às gasolinas não aditivadas.

\section{CONCLUSÕES}

O método demonstrou que o enriquecimento decorrente da concentração dos aditivos no resíduo do ensaio de destilação, que é um ensaio de rotina nos laboratórios de controle de qualidade de combustíveis, foi satisfatório para a quantificação dos aditivos detergentes dispersantes somente por TG.

O aditivo detergente dispersante utilizado A apresentou o mesmo comportamento termogravimétrico, do que outros aditivos comerciais analisados anteriormente pelos autores, assim como o resíduo de destilação de gasolina com aditivo presente.

O método apresentou resultados satisfatórios com respeito ao erro relativo em relação aos valores nominais utilizados na preparação das misturas, pois o erro relativo máximo obtido foi de $9 \%$.

O método é mais simples e de menor custo em relação aos até então encontrados na literatura e os resultados apontam como potencialmente adequado para análises de quaisquer gasolinas aditivadas, independentemente dos seus pacotes de aditivos, sendo, portanto, passível de tornar-se um método de referência, aplicável pelos laboratórios de controle de qualidade de combustíveis.

\section{REFERÊNCIAS}

[1] AGÊNCIA NACIONAL DE PETRÓLEO, GÁS NATURAL E BIOCOMBUSTÍVEIS ANP. Resolução ANP N 40 de 25 de Outubro de 2013 (DOU 28 de Outubro de 2013; Republicada DOU 30 de Outubro de 2013). Disponível em http://www.anp.gov.br. Acesso em 08 de abril de 2016.

[2] NIKITINA, E.A.; EMEL'YANOV, V.E.; KRYLOV I. F.; FEDOROVA, A. V. Detergent additives to automotive gasolines. Chemistry and Technology of Fuels and Oils, v. 42, n. 1, p. 30-34, 2006.

[3] BRITO, L. R. Determinação de aditivos detergentes e dispersantes em gasolina utilizando a técnica do ring oven e imagens hiperespectrais na região do infravermelho próximo. Dissertação. Instituto de Química, Universidade Federal de Pernambuco, Pernambuco, 2014.

[4] SILIN, M. A.; IVANOVA, L. V.; BUROV, E. A.; KOSHELEV, V. N.; BORDUBANOVA, E.G. Synthesis and Testing of Polyalkenyl Succinimides as Components of Detergent Additives for Motor Fuels. Petroleum Chemistry, v. 52, n. 4, p. 272-277, 2012.

[5] NADIM, F.; ZACK, P.; HOAG, G.E.; LIU, S. United States experience with gasoline additives. Energy Policy, v. 29, n. 1, p. 1-5, 2001. 
[6] DANILOV, A.M. Progress in Research on Fuel Additives (Review). Petroleum Chemistry, v.55, n. 3, p. 169-179. 2015.

[7] MEDEIROS, A.R.B. Uso de ATR/FTIR e FTNIR associado à técnicas quimiométricas para quantificação de aditivos em gasolina automotiva. Dissertação. Instituto de Química, Universidade de Brasília, Brasília, 2009.

[8] KARPOV, S. A. Improving the environmental and performance properties of automotive gasolines. Detergent additives. Chemistry and Technology of Fuels and Oils, v. 43, n. 3, p. 173-178, 2007.

[9] COLAIOCCO, S.; LATTANZIO, M. Determination of additives in gasoline by SEC coupled to a light-scattering detector - Chemometrics Application. Journal High Resolution Chromatography, v. 18, n. 1, p.387-388. 1995.

[10] GRAPNER, O.; MUNDT, M.; SCHUTZE, A.; LOUIS, J.J.J.; KENDALL, D.R.; TAIT, N.P. Gasoline additives. US 20050172545A1. 2005.

[11] SILVA, M. P. F.; BRITO, L. R.; HONORATO, F. A.; PAIM, A. P. S.; PASQUINI, C.; PIMENTEL, M. F. Classification of gasoline as with or without dispersant and detergent additives using infrared spectroscopy and multivariate classification. Fuel, v. 116, n. 1, p. 151-157, 2014.

[12] PIMENTEL, M. F.; HONORATO, F. A.; PASQUINI, C.; ROHWEDDER, J.J.R.; SILVA, M.P.F.; BRITO, L.R. Determination of detergent and dispersant additives in gasoline by ring oven and near infrared hypespectral imaging. Analytica Chimica Acta, v. 863 , n. 1, p. 9-19, 2015.

[13] SANTOS, A.P.F. Desenvolvimento de métodos para separação e identificação semiquantitativa do biodiesel e/ou óleo vegetal no óleo diesel e de aditivos em gasolina. Dissertação. Escola de Química, Universidade Federal do Rio de Janeiro, Rio de Janeiro, 2012.

[14] SANTOS, A. P. F.; SILVA, K. K.; DWECK, J.; D'AVILA, L. A. Quantification of Detergent-dispersant Additives in Gasoline by size-exclusion Chromatography and Thermogravimetry. Fuel, v.194, n. 1, p. 166-170, 2017.

[15] SANTOS, A. P. F.; SILVA, K. K.; DWECK, J.; D’AVILA, L. A., Characterization of Fuel Detergent-Dispersant Additives by Thermogravimetry, J Therm. Anal. Cal. V131, 1, p78-788 2018.

[16] SILVA, D. P. Análise de gasolina aditivada por espectrometria de massas e cromatografia líquida com espectrometria de massas sequencial. Dissertação. Faculdade de Engenharia Mecânica, Universidade Estadual de Campinas, São Paulo, 2017.

[17] PINTO, V. S.; GAMBARRA-NETO, F. F.; FLORES, I. S.; MONTEIRO, M. R.; LIÃO, L.M. Use of $1 \mathrm{H} \mathrm{NMR}$ and chemometrics to detect additives present in the Brazilian commercial gasoline. Fuel, v. 182, n. 1, p. 27-33, 2016.

[18] SOUZA, C.G.; D’AVILA, L.A. Processo para detecção de aditivos básicos em combustíveis e óleos lubrificantes e kit para realizar o referido processo. BR1020120292300 - WO2014/075158A1. 2014. 
[19] AMERICAN SOCIETY FOR TESTING AND MATERIALS. ASTM D86. Standard Test Method for Distillation of Petroleum Products at Atmospheric Pressure. 2017.

[20] LIN, J.-J.; WU, J.-J.; SHAU, S.-M.; HO, Y.-S. Thermal Stability and Combustion Behaviors of Poly(oxybutylene)amides. Polymer Journal, v. 34, n. 2, p. 72-80, 2002.

[21] DWECK, J. Fatores experimentais importantes em termogravimetria. Publicação interna do curso de análises térmicas de materiais e processos. Escola de Química, Universidade Federal do Rio de Janeiro, Rio de Janeiro, 2015

[22] IONASHIRO, M. G.; CAIRES, F. J.; GOMES, D. J. C. GIOLITO: Fundamentos da termogravimetria e análise térmica diferencial/ calorimetria exploratória diferencial. $2^{a}$ Edição, São Paulo. Giz Editorial. 2014, p. 18.

[23] INSTITUTO NACIONAL DE METROLOGIA, QUALIDADE E TECNOLOGIA INMETRO. Orientação sobre Validação de Métodos Analíticos. DOC-CGCRE-008, 2016. Disponível em http://www.inmetro.gov.br/Sidoq/Arquivos/Cgcre/DOQ/DOQCgcre-8_05.pdf. Acesso em 02 de fevereiro de 2018. 